\title{
DETERMINAÇÃO DAS DIMENSÕES DE UM BIODIGESTOR EM FUNÇÃO DA PROPORÇÃO GÁS/FASE LÍQUIDA
}

\author{
Deyvid da Silva Ribeiro \\ Engenheiro Agrônomo, com ênfase em Manejo dos Recursos Naturais, Técnico \\ Agropecuário, Especialista em Administração de Projeto. Mestrando em Gestão Ambiental de \\ Empresas. E-mail: deyvidribeiro@ gmail.com
}

\section{RESUMO}

Devido ao risco de contaminação ambiental através da má manipulação dos excrementos animais produzidos pela pecuária, faz-se necessário buscar alternativas eficientes e de baixo custo para resolver este problema. Entre as diversas soluções, a FAO recomenda o Biodigestor como uma alternativa viável e prática. Como benefícios da utilização dos biodigestores, destacam-se a produção de biogás, descontaminação biológica e química dos dejetos animais e a geração de biofertilizante através de processo anaeróbico. O objetivo deste trabalho foi determinar um modelo para calcular as dimensões de um biodigestor tubular em função da proporção gás/fase líquida desejada. A metodologia utilizada foi baseada nas recomendações de Ribeiro para desenhos de biodigestores, o Raciocínio Lógico-Matemático e os programas EXCEL 2007® e AutoCAD 2009® que foram utilizados para auxiliar nos cálculos e nas simulações de campo. Os modelos encontrados demonstraram $100 \%$ de exatidão no que se refere ao dimensionamento e $100 \%$ de aplicabilidade em campo para biodigestores com proporções gás/fase líquida menores a $40 \%$.

PALAVRAS CHAVE: Biodigestor, modelo, dimensões, excrementos animais e contaminação.

\section{DETERMINACIÓN DE LAS DIMENSIONES DE UN BIODIGESTOR EN FUNCIÓN DE LA PROPORCIÓN GÁS/FASE LÍQUIDA}

\section{RESUMEM}

Debido al riesgo de contaminación ambiental ocasionada por la mala manipulación de las excretas animales generada por la pecuaria, se ha creado la necesitad de buscar alternativas eficientes y de bajo costos para solucionar ese problema. Entre las diversas soluciones, FAO recomienda el Biodigestor como una alternativa viable y práctica. Entre los beneficios generados por el biodigestor, se destacan: la producción de biogás, la descontaminación biológica y química de las aguas servidas y la producción de bioabono Con el objetivo de determinar un modelo para calcular las dimensiones de un biodigestor tubular en función de la proporción gas/fase líquida, se realizó el presente trabajo. Para ello, fueron utilizados las recomendaciones de Ribeiro, el Raciocinio Lógico-Matemático y los programas EXCEL 2007® y AutoCAD 2009® para auxiliar con los cálculos y en las simulaciones de campo. Los modelos demostraron $100 \%$ de exactitud para el dimensionamiento y $100 \%$ de aplicabilidad en campo para casos de biodigestores con proporciones gas/mescla líquida menores a $40 \%$.

PALABRAS CLAVES: Biodigestor, modelo, dimensiones, excretas de animales y contaminación. 


\section{DETERMINAÇÃO DAS DIMENSÕES DE UM BIODIGESTOR EM FUNÇÃO DA PROPORÇÃO GÁS/FASE LÍQUIDA}

\section{INTRODUÇÃO}

Um dos problemas da pecuária é a produção de excrementos animais. Quando mal manipulados, estes podem contaminar os aquífferos, o solo e as pessoas, pois os excrementos animais contêm bactérias patogênicas, fosfatos, nitratos e matéria orgânica em quantidades excessivas (VÁZQUEZ e MANJARREZ, 1993). O biodigestor é uma alternativa barata e eficiente para resolução dessa problemática, já que através da fermentação anaeróbica ou biodigestão é possível reduzir até $85 \%$ dos coliformes fecais e $95 \%$ dos fungos (MARCHAIM, 1992) contidos nos dejetos animais com possibilidades de biofermentação, e por sua vez se pode produzir biogás e biofertilizantes (LUCAS JUNIOR e SILVA, 1998; BROWN, 1987). O biogás é uma fonte renovável de energia utilizada como gás doméstico, para geração de eletricidade ou calefação (OLIVEIRA, 2005). O biofertilizante é usado como adubo orgânico para aplicação direta ao campo (IICA, [2008]) ou servir de matéria-prima para o preparo de compost ou bokashi.

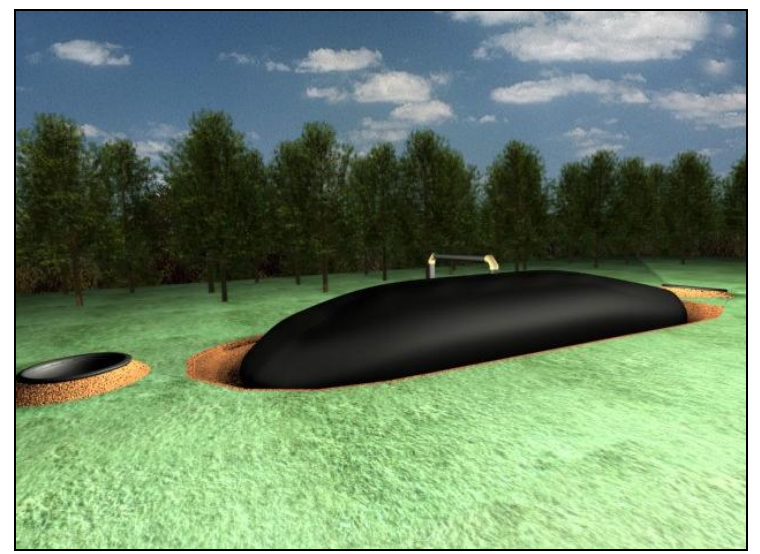

Figura 1. Ilustração de um biodigestor tubular (Oliveira, 2005).

Segundo Ribeiro (2004), o biodigestor tubular é dividido em duas partes: uma para a fase líquida e outra para a fase de gás. Isto é, dentro do biodigestor existe na parte superior, uma área denominada campana, destinada para reservar o biogás produzido pela fermentação anaeróbica e na parte inferior uma área denominada fossa, destinada à mistura líquida, (excremento animais mais água) que entra no sistema (BOTERO e PRESTON, 1986). O autor afirma que é comum que a proporção gás/fase líquida seja alterada segundo a necessidade do projeto de instalação do biodigestor, ou seja, do tempo de retenção da fase líquida, da quantidade de matéria-prima disponível e do consumo de gás diário.

O objetivo deste trabalho é apresentar um modelo matemático para calcular, em função da proporção gás/fase líquida desejada, as dimensões de um biodigestor tubular de plástico. 


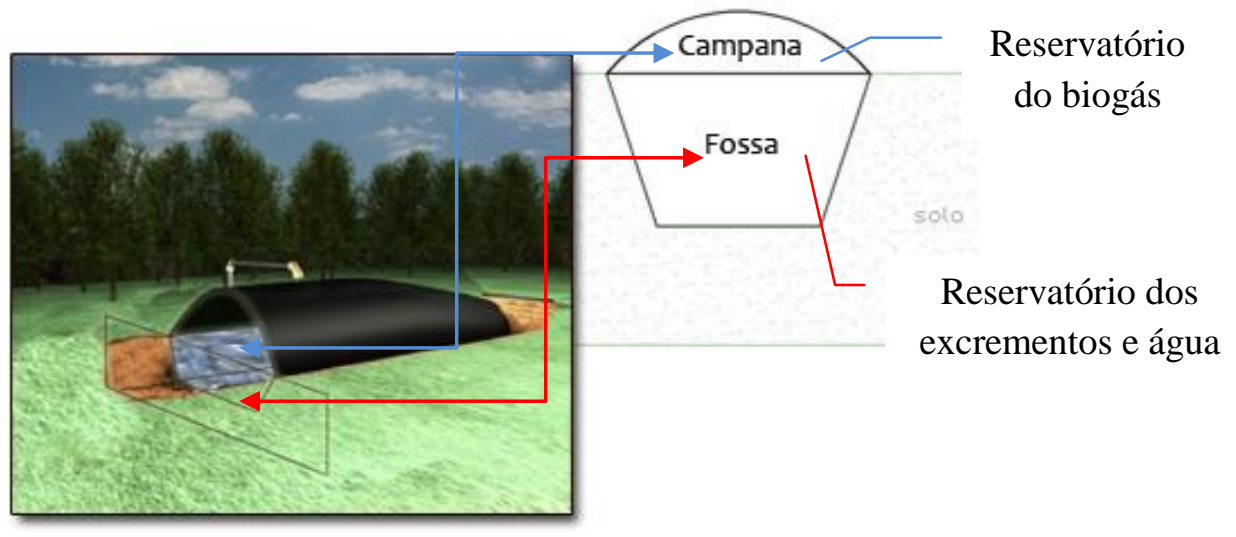

\section{Figura 2. Corte transversal de um biodigestor tubular (Adaptado de OLIVEIRA, 2005).}

\section{REVISÃO DA LITERATURA}

$\mathrm{O}$ uso do biodigestor é anterior ao século XX. Na Inglaterra, o biogás já era usado para iluminação pública (BROWN, 1987). Alguns historiadores apontam a China ou a Índia como países de origem da tal tecnologia (CRISTIANA et al, 1997).

O biogás é produzido através da fermentação anaeróbica no interior do biodigestor (LETTINGA e VAN HAANDEL, 1993). O processo de fermentação anaeróbica ou digestão libera a energia química contida na matéria orgânica, em forma de biogás, cujos principais componentes são: gás metano $\left(\mathrm{CH}_{4}\right)$ e o dióxido de carbono $\left(\mathrm{CO}_{2}\right)$ (CIPAV, [2004]). O efluente produzido, denominado biofertilizante, tem um alto conteúdo de nutriente e baixo conteúdo de bactérias patogênicas (BROWN, 1987 e MARCHAIM, 1992), pois durante a fermentação se conservam todos os nutrientes da matéria-prima, liberando em forma de gás $\left(\mathrm{CH}_{4}, \mathrm{CO}_{2}, \mathrm{H}_{2} \mathrm{~S}\right.$ entre outros) somente de $5 \%$ a $10 \%$ do volume total dos excrementos animais utilizados (IICA, [2008]).

As altas temperaturas alcançadas durante a biodigestão (ACUÑA, 1998) e os ácidos produzidos na fermentação eliminam até $90 \%$ das bactérias patogênicas (NIMUKUNDA, 2003). Por outro lado, Fregoso et al (2001) conseguiram eliminar 100\% dos coliformes fecais num processo de digestão de 50 dias.

Os dados demonstrados anteriormente são comprovados por Pedraza et al (2002) ao corroborar que a biodigestão reduz em até $92 \%$ da Demanda Biológica de Oxigênio (DBO) e por Fregoso et al (2001) ao comprovarem uma redução de 47\% da Demanda Química de Oxigênio (DQO) da matéria orgânica contida na fase líquida.

O uso do biodigestor também traz benefícios econômicos e ambientais, pois reduz os custos de consumo de gás, eletricidade, combustíveis fósseis e reduz a dependência de fertilizantes inorgânicos. Ademais, os benefícios econômicos gerados por esse método crescem ano a ano após a sua instalação, sendo que nos primeiros meses, o produtor já recupera o investimento inicial usado para a instalação do biodigestor tubular (BOTERO e AGUILAR, 2006).

Segundo a sua forma de operação, os biodigestores se classificam em: de regime continuo; de regime semi-continuo; de regime estacionário, e de movimento horizontal (FREGOSO et al, 2001). 
Para os três primeiros biodigestores, os cálculos de dimensionamentos utilizam técnicas matemáticas não triviais, pois é necessário otimizar funções não lineares restritas.

Para auxiliar os produtores rurais e outros profissionais durante os cálculos, Portes e Florentino (2006) desenharam um aplicativo computacional para dimensionar tais biodigestores. Entretanto, o calculo de dimensionamento dos biodigestores de movimento horizontal, conhecidos também por biodigestor tubular, é mais fácil.

O uso de um biodigestor tubular foi registrado pela primeira vez em 1957, na República da África do Sul (GUNNERSON et al, 1989). Como o custo de instalação ainda era elevado, Preston e Rodriguez ([2002]) desenvolveram um biodigestor tubular usando plástico de polietileno de baixo custo, baixo peso e de igual eficiência ao seu antecessor. O primeiro protótipo desse biodigestor de baixo custo foi provado por uma família de Etiópia em 1985 (BOTERO E AGUILAR, 2006). Atualmente o biodigestor demonstrado na Figura 3 é muito utilizado na América Central e do Norte, Colômbia, Equador, Venezuela e alguns países africanos e asiáticos. Mesmo denominado biodigestor tubular, são instalados em fossas quadradas, retangulares ou trapezoidais.

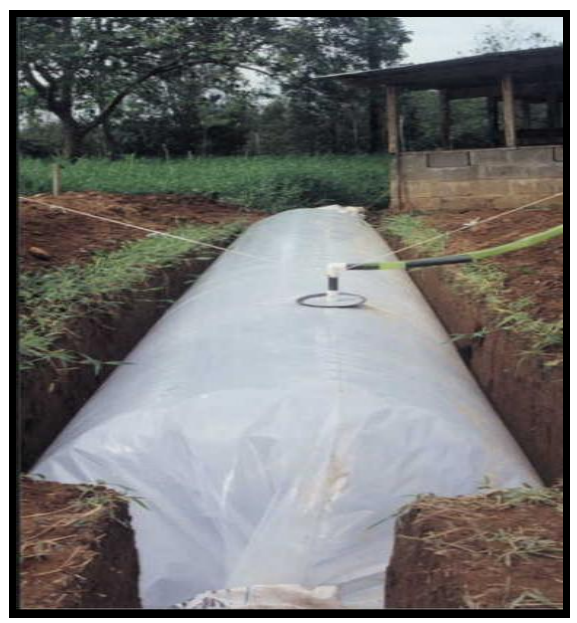

Figura 3: Biodigestor tubular

(Pastoral Social, Limón, Costa Rica mencionado por Víquez et al, 2003).

\section{MATERIAIS E MÉTODOS}

Este é um estudo matemático. Por tanto, a metodologia utilizada foi feita baseando-se em um Modelo conceitual apoiado na Análise de programas computacionais. A seguir é detalhado o processo para o Modelo Conceitual e a Análise do modelo.

\section{MODELO CONCEITUAL}

Os parâmetros utilizados na estruturação dos modelos conceituais (Figura 4) foram baseados nas sugestões de Ribeiro (2004), pois o mesmo desenvolveu diferentes modelos de biodigestores tubulares de plásticos com fossas trapezoidais utilizados atualmente por pequenos produtores na América Central e por uma grande cooperativa de Produtos Lácteos em Costa Rica, denominada Dos Pinos.

O desenvolvimento das equações foi realizado com base no Raciocínio Lógico-Matemático conforme preceitua Ribeiro (2004) e Larson et al (1999). 
Os ajustes foram feitos através de tentativa e erro, ou seja, são atribuídos valores para os parâmetros iniciais para testar os modelos, sendo refeito cálculos até que seja satisfeito o objetivo do trabalho.

Para iniciar o modelo conceitual, buscou-se relacionar os parâmetros iniciais detalhados na figura 4: Arco, Lateral $(b)$, Altura $(h)$, Base $(b)$, Largura maior $(a)$ com a proporção gás/fase líquida desejada. Logo, relacionar quanto do corte transversal da bolsa de plástico será destinada à formação do Arco da campana (Figura 2 e 4) para as possíveis proporções gás/fase líquida. Com as informações definidas anteriormente, já é possível realizar o processo de definição das equações para calcular as dimensões da fossa ou fase líquida.

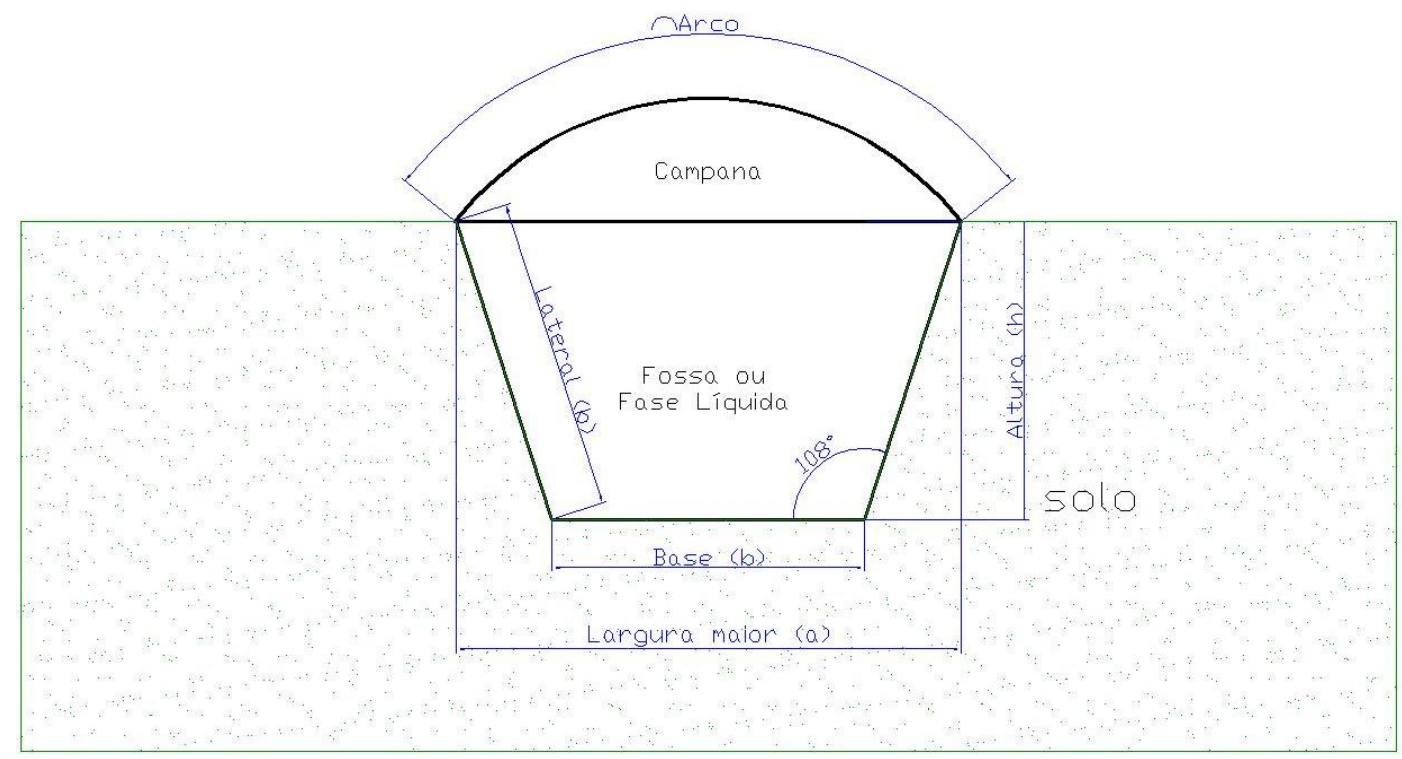

Figura 4. Parâmetros bases utilizados nos modelos (Autoria própria, 2009).

\section{ANÁLISES DOS MODELOS}

Os modelos foram testados usando dois programas: Excel 2007® e o AutoCAD 2009®. Em Excel 2007®, se calcularam todas as variações possíveis de dimensões que podem ocorrer em função da proporção gás/fase líquida desejada, ou seja, de $0 \%$ até 99,99\% (RIBEIRO, 2004). Provando desta forma a exatidão dos modelos através de regressões. No AutoCAD 2009® foram simuladas 200 variações possíveis de dimensões calculadas pelos modelos, provando assim a sua aplicabilidade em campo. As variações de dimensões usadas nas simulações foram tomadas aleatoriamente entre $0 \%$ a $99,99 \%$ de proporção gás/fase líquida.

\section{RESULTADOS E DISCUSSÃO MODELOS}

Após a fase de desenvolvimento dos Modelos Conceituais, foi montada uma tabela com as equações definidas para determinação das dimensões de um biodigestor tubular de plástico.

As equações demonstradas na tabela foram testadas com Excel 2007® para simular todas as possíveis proporções gás/fase líquida. Os resultados obtidos demonstraram que é possível instalar biodigestores com $0 \%$ até $99,99 \%$ de proporção gás/fase líquida. 
Por outro lado, nas simulações realizadas no AutoCAD 2009® se obteve 100\% de exatidão, no que se refere à proporção gás/fase líquida. Entre tanto, a experiência do autor e de Botero (2008) em campo, há demonstrado que não é pratico e viável por condições física, uma instalação de biodigestores com proporção "p" maiores a $40 \%$ [Eq. 2], pois o volume da fase líquida nestas condições não será suficiente para manter a campana cheia de gás, ou seja, elevada dentro do período de retenção, fazendo com que a campana colapse sobre a fase líquida.

Tabela: Fórmulas (modelos) para determinar as dimensões do biodigestor.

\begin{tabular}{|c|c|}
\hline Fórmula & Descrição \\
\hline 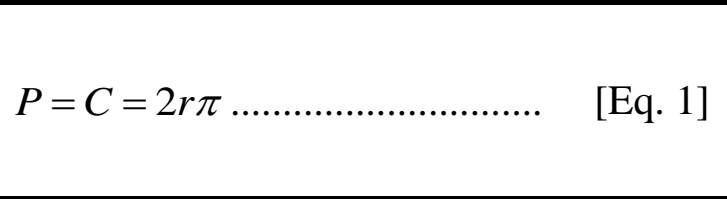 & $\begin{array}{l}P=\text { Perímetro total transversal (campana mais } \\
\quad \text { fossa) } \\
C=\text { Circunferência da bolsa. } \\
r=\text { raio da bolsa plástica. }\end{array}$ \\
\hline$A_{\%}=0,621 p^{2}-0,042 p+0,352$ & $\begin{aligned} A_{\%}= & \text { Porcentagem do perímetro transversal } \\
& \text { destinado ao arco. } \\
p= & \text { Proporção da fase gasosa desejada }(p \leq 0,4) .\end{aligned}$ \\
\hline 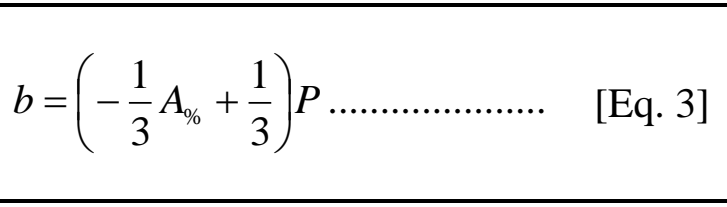 & $\begin{array}{l}b=\text { Lateral, base ou largura menor da fossa. } \\
A \%=\text { Porcentagem do perímetro transversal } \\
\quad \text { destinado ao arco. } \\
P=\text { Perímetro total transversal. }\end{array}$ \\
\hline$a=1,618 b \ldots \ldots \ldots \ldots \ldots \ldots \ldots$ & $\begin{array}{l}a=\text { Largura maior da fossa. } \\
b=\text { Lateral, base ou largura menor da fossa. }\end{array}$ \\
\hline$h=0,951 b \ldots \ldots \ldots \ldots \ldots \ldots$ & $\begin{array}{l}h=\text { Profundidade da fossa. } \\
b=\text { Lateral, base ou largura menor da fossa. }\end{array}$ \\
\hline$A_{f}=0,4755(a+b) b \ldots \ldots \ldots \ldots \ldots \ldots \ldots \ldots \ldots \ldots \ldots$ & $\begin{array}{l}A_{f}=\text { Área transversal da fossa } \\
a=\text { Largura maior da fossa. } \\
b=\text { Lateral, base ou largura menor da fossa. }\end{array}$ \\
\hline 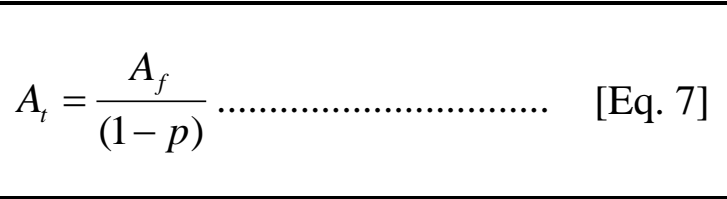 & $\begin{array}{l}A_{t}=\text { Área total transversal ou área transversal } \\
\text { da fossa mais área transversal da campana. } \\
A_{f}=\text { Área transversal da fossa. } \\
p=\text { Proporção da fase gasosa desejada. }\end{array}$ \\
\hline$A_{g}=A_{t}-A_{f} \cdots \cdots$ & $\begin{array}{l}A_{g}=\text { Área transversal da campana ou área } \\
\text { transversal destinada para o gás. } \\
A_{t}=\text { Área total transversal ou área transversal } \\
\text { da fossa mais área transversal da campana } \\
A_{f}=\text { Área transversal da fossa. }\end{array}$ \\
\hline 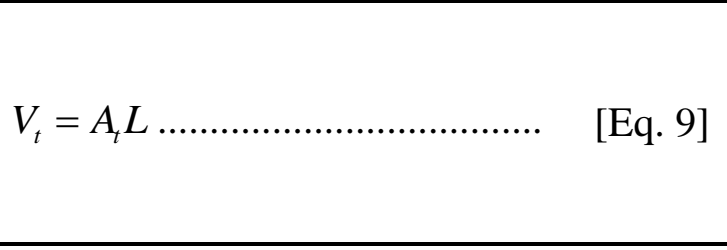 & $\begin{array}{l}V_{t}=\text { Volume total do biodigestor (campana } \\
\text { mais gás). } \\
A_{t}=\text { Área total transversal ou área transversal } \\
\text { da fossa mais área transversal da campana. } \\
L=\text { Comprimento do biodigestor. }\end{array}$ \\
\hline ..................... [Eq. 10] & $\begin{array}{l}V_{f}=\text { Volume total da fossa. } \\
A_{f}=\text { Área transversal da fossa. } \\
L=\text { Comprimento do biodigestor. }\end{array}$ \\
\hline 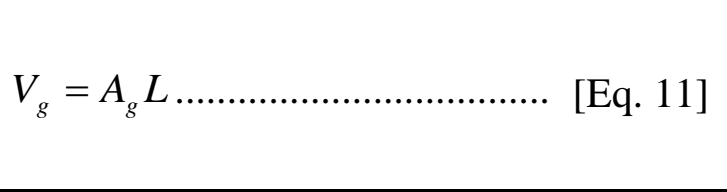 & $\begin{array}{l}V_{g}=\text { Volume total de gás. } \\
A_{g}=\text { Área transversal da campana ou área } \\
\quad \text { transversal destinada para o gás. } \\
L=\text { Comprimento do biodigestor. }\end{array}$ \\
\hline
\end{tabular}




\section{CONCLUSÕES}

As equações desenvolvidas neste trabalho mostram que é possível criar um modelo matemático para calcular as dimensões de um biodigestor tubular de plástico em função da proporção gás/fase líquida entre $0 \%$ a 99,99\%.

O modelo matemático desenvolvido neste trabalho não se aplica para construção em campo de biodigestores tubulares de plásticos com proporções gás/fase líquida maiores a $40 \%$.

\section{REFERÊNCIAS}

1. ACUÑA, J. F. Diseño y análisis financiero de una finca integra, enfocada al ecoturismo. 1998. 105 f. Trabalho de conclusão de curso (Bacharelado em Agronomía), Universidade EARTH, Guácimo, 1998.

2. AUTOCAD 2009®. AutoCAD Land Desktop 2009. Autodesk, Inc. 2008.

3. BOTERO, R. Biodigestor: comentarios sobre las dimensiones de la campana y la fosa [mensagem pessoal]. Mensagem recebida por <deyvidribeiro@gmail.com> em 08 de ago. 2008.

4. BOTERO, R.; PRESTON, T.R. Manual de instalación de un biodigestor a bajo costo. Cali, 1986, $35 \mathrm{p}$.

5. BOTERO, R; AGUILAR, F.X. Estimación de los Beneficios Económicos Totales de la producción de biogás utilizando un biodigestor de polietileno de bajo costo. Tierra Tropical, Universidad EARTH, v. 2, n. 1, p 15-25. 2006.

6. BROWN, N. Biogas systems in development. Appropriate technology. [S.L.: s.n.], 1987. v.14, n.3, p. 5-7. 1987.

7. CIPAV. Centro de Investigación en Sistemas Sostenibles de Producción Agropecuaria, Cali, Colombia. Disponível em http://www.cipav.org.co, [2004]. Acesso em 10 de out. 2005.

8. CRISTIANA et al. Biodigestor. Disponível em http://www.cdcc.sc.usp.br/escolas/ juliano/biodiges.html, 1997. Acesso em 6 abr. 2004.

9. EXCEL 2007®. Microsoft Office Excel 2007. Parte do Microsoft Enterprise 2007. Microsoft Corporation. 2008.

10. FAO. Biodigestor de plástico de flujo continuo, generador de gás y bioabono a partir de águas servidas. CIPAV: Fundación Centro para la Investigación em Sistemas Sostenibles de Producción Agropecuaria. Ciudad de Guatemala, 1995, 17 p.

11. FREGOSO, M. J. S. et al. Producción de biofertilizantes mediantes biodigestion de la excreta Liquida de cerdo. Revista Terra Latino Americana, Universidad Autonoma Chapingo, Texcoco, v. 19, n.4. p 353-362. Set. 2001.

12. GUNNERSON, C. G. et al. Anaerobic digestion (biogas) systems: principles of integrated use and their application in developing countries. In: WISE, D.L. International biosystems. Boca Raton: CRC Press, 1989, v.1, 340 p. il.

13. IICA. Instituto Interamericano de Cooperación para la Agricultura. Nicarágua. Disponível em http://www.iica.int.ni/estudios_pdf/biodigestores.pdf, [2008]. Acesso em 14 Out. 2009. 
14. LARSON, R. E. et al. Cálculo y Geometría Analítica. Nueva York. Ed. Mc Graw Hill. $6^{\circ}$ ed. 887 p. 1999.

15. LETTINGA, G; VAN HAANDEL, A. Anaerobic digestion for energy production and environmental protection. In: Johansson, T. et al (ed.). Renewable Energy: Sources for fuels and electricity. Earthscan: Island Press, 1993, p. 817-841.

16. LUCAS JÚNIOR, J.; SILVA, F.M. Aproveitamento de resíduos agrícolas para a geração de energia. In: CONGRESSO BRASILEIRO DE ENGENHARIA AGRÍCOLA, 27., 1998. Poços de Caldas. Anais... Lavras/Mg: UFLA, SBEA, 1998. p. 63-67.

17. MARCHAIM, U. Biogas processes for sustainable development. In: FAO Agricultural Services Bulletin, 95., 1992. 232 p.

18. NIMUKUNDA, F. Análisis de las aguas servidas en los biodigestores de la Finca Pecuaria Integrada de la Universidad EARTH. 2003. 63 p. Trabalho de conclusão de curso (Bacharelado em Agronomía), Universidade EARTH, Guácimo, 2003.

19. OLIVEIRA, L. R. P. Biodigestor. In: VII Simpósio Goiano de Avicultura e II Simpósio Goiano de Suinocultura - Avesui Centro-Oeste, 2005. Goiânia/GO, 2005. p. 4-8.

20. PEDRAZA et al. Evaluación de los biodigestores en geomembrana (pvc) y plástico de invernadero en clima medio para el tratamiento de aguas residuales de origen porcino. Livestock Research for Rural Development. v. 14, n. 1, 2002. Disponível em http://www.cipav.org.co/Irrd14/1/pedr141.htm. Acesso em 10 nov. 2004.

21. PORTES, Z. A.; FLORENTINO, H. O. Aplicativo computacional para projetos e construções de biodigestores rurais. Energia Agrícola, Botucatu, São Paulo, v. 21, n. 1. p 118-138. 2006.

22. PRESTON, T. R; Rodriguez, L. Biodigestor: Manual de Instalação. Ho Chin Minh City: University of Tropical Agriculture Foundation, [2002]. 7 p.

23. RIBEIRO, D. Biodigestor: Maximización del volumen. Universidade EARTH, Guácimo. 2004. 13 p. Trabalho de aluno.

24. VÁZQUEZ, B., MANJARREZ, R. 1993. Contaminación del agua subterránea por la actividad porcícola. Tecnología del Agua, Barcelona, v. 109. p 38-43.

25. VÍQUEZ, C. et al. Manual Práctico para la Implementación de un Sistema de Descontaminación Productiva de Aguas Servidas en un Sistema Pecuario. Las Mercedes de Guácimo: Universidad EARTH, 2003, 15 p. 